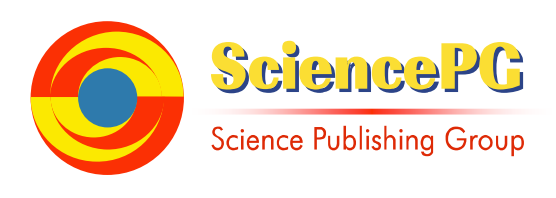

\title{
Discussion on the Accommodation Infection Prevention-control of the Supportive Medical Team Assigned to Hubei During COVID-19 Outbreak
}

\author{
Quan Jiang", Jiewei Huang ${ }^{2, *}$, Xianzhong $\mathrm{Wu}^{3}$, Xiaohong $\mathrm{Mo}^{4}$, Piao $\mathrm{Ye}^{5}$ \\ ${ }^{1}$ Department of Hepatobiliary Surgery, the First Affiliated Hospital of Jinan University, Guangzhou, China \\ ${ }^{2}$ Department of Nursing, the First Affiliated Hospital of Jinan University, Guangzhou, China \\ ${ }^{3}$ Department of Intervention Therapy, the First Affiliated Hospital of Jinan University, Guangzhou, China \\ ${ }^{4}$ Department of Cardiovascular Intensive Care Unit, the People's Hospital of Gaozhou, Maoming, China \\ ${ }^{5}$ Department of the Infection Control Section, Guangzhou First People's Hospital, Guangzhou, China
}

Email address:

151733060@qq.com (Quan Jiang),dmapn@126.com (Jiewei Huang)

*Corresponding author

\section{To cite this article:}

Quan Jiang, Jiewei Huang, Xianzhong Wu, Xiaohong Mo, Piao Ye. Discussion on the Accommodation Infection Prevention-control of the Supportive Medical Team Assigned to Hubei During COVID-19 Outbreak. American Journal of Nursing Science.

Vol. 9, No. 5, 2020, pp. 359-364. doi: 10.11648/j.ajns.20200905.18

Received: September 5, 2020; Accepted: September 21, 2020; Published: September 28, 2020

\begin{abstract}
Objective To summarize the infection prevention and control experiences in the resident of medical team giving support to Wuhan, and to provide some reference for our same occupations. Methods Based on the previous experience of supporting Wuhan medical teams and combined with the actual situation of our own medical team, we carried out the prevention and control of resident infection from six aspects, including the establishment of infection control team, training for control knowledge, resident layout, standardizing entry and exit processes, personnel behavior management, personnel health management. Results By the time of April 10, 2020, 172 members of our medical team had been fighting in Wuhan for 50 days and nights, in the face of the COVID-19 public health emergency, the medical team protected the members and there were no cases of medical staff infection. Conclusion In the novel coronavirus pneumonia affected area, it is effectively to prevent the occurrence of infection and personal infection in medical teams through a series of feasible prevention and control measures. At the same time, we found that many members of the medical team had mental health problems. Early psychological crisis intervention training is helpful for medical team members to better fight against the epidemic.
\end{abstract}

Keywords: Novel Coronavirus, Novel Coronavirus Pneumonia, Resident of Medical Team, Infection Prevention and Control, Experience

\section{Introduction}

COVID-19 is a kind of highly contagious, acute respiratory disease. The main routes of transmission are via respiratory droplets and direct contact, and there is a possibility of aerosol transmission $[1,2]$. To combat the contagion, the Chinese government has implemented community-wide containment strategies such as home quarantine, business and public transportation shutdown, and class suspension for all schools. Medical personnel have become the main force in medical treatment and pandemic control. The outbreak of COVID-19 pandemic has resulted in a surge in the number of patients requiring treatment, which markedly exceeds the upper limit of medical treatment capacity in Hubei Province, China, especially in Wuhan City.

In order to assist in the treatment of patients in Hubei Province, China, medical rescue teams from all over the country went to Hubei to provide medical support. Because of their close contact with patients, medical staff is at high-risk of contracting the disease, and spreading it to others. Infection of a single person on the medical staff can lead to a chain of infection. To avoid an infection outbreak of the medical staff, strong measures are needed to prevent 
infection. This is an important measure to avoid cross-infection and reduce the risk of spreading the disease.

The purpose of this report is to summarize the infection prevention and control methods used by our medical team in Wuhan, China.

\section{Establishment of an Infection Control and Supervision Team}

COVID-19 has been categorized as a Class B infectious disease, and the management of Class A infectious diseases has been adopted for it. To protect the safety of the medical team and reduce the risk of infection, we adhered to an "infection control first" policy for medical care. Our medical team was composed of 172 members, of which 126 (73\%) were nursing staff, from 6 hospitals in Guangdong Province. Upon arrival in Wuhan, an infection control and supervision team of 11 persons was established, with a team leader and a deputy team leader. The team leader was a physician with training in infection control, and the deputy team leader was an infection control nurse specialist. The other 9 team members were nurses with experience in infection control, who were highly responsible, strict, courageous, and careful.

\section{Infection Control Knowledge Training}

A survey of infection control knowledge [3] found that $31 \%$ of respondents believed they did not have sufficient knowledge of personal and patient infection control, especially isolation methods for infection control and cleaning and disinfection of the environment. At the same time, the medical team had some misunderstandings with respect to certain factors related to the spread of infection, such as air conditioning, outerwear, and shoes. In addition, they were not aware that spraying alcohol or chlorine disinfectants when entering or leaving the hotel room was not a correct method of disinfection.

In view of this, and according to the "Notice of the General Office of the National Health Commission on Issuing the Prevention and Control Protocols for CoVID-19 (Fifth Edition)" (Disease Control and Protection Letter issued by National Health Office [2020] No. 156) [4], and the Guangdong medical team's experience in early anti-epidemic work in Hankou Hospital of Wuhan City, the members of the Guangdong Provincial Medical Team received rigorous training and assessments after arriving in Wuhan. The training included online and offline trainings and assessment, and members were required to pass an assessment test before beginning work.

Online trainings were mainly conducted through a WeChat group, and used Power Point presentations, recorded video, and live video conferences. The content of the training included the epidemiological characteristics of the new coronavirus, diagnosis and treatment of CoVID-19, prevention and control protocols for the new coronavirus, methods for putting on and taking off protective equipment, and patient management. After completion of the online training, the staff members were assess by a questionnaire. Members were allowed to take the assessment 3 times, and a score over 90 was required for qualification. After each time a member took the assessment a session was conducted with explanations for wrong answers, which helped to strengthen and consolidate their knowledge.

Offline training and assessment was similar to that of the online training, but focused on putting on and removal of personal protective equipment, infection prevention and control schemes, cleaning and disinfection processes, the contents of different types of disinfectant solutions, and partition specifications of rooms with different layouts. A score of over 95 for the assessment of putting on and removing personal protective equipment was required for qualification. The pass rate of the team members was $100 \%$.

\section{Hotel Layout and Environmental Management}

\subsection{Specific Entrance and Exit Accesses Were Defined at the Hotel}

The team members could only leave the hotel through defined exits, and enter the hotel through defined entrances. Paper towel, Hands-free disinfectants, disinfectant foot pads, and foot-operated special trash cans were placed at the entrance. When returning to the hotel from the hospital, team members disinfected their hands first, followed by replacing their masks and caps with new ones, disinfecting their hands again, stepping on the disinfected foot pads, and finally receiving a body temperature measurement by the hotel staff. Members could only enter the hotel if their body temperature was normal.

\subsection{Elevator Management}

There were 6 elevators in the hotel. Three elevators (left side) were defined as "clean" elevators and used for team members to leave the hotel. The other 3 elevators (right side) were defined as "non-clean" elevators and used for team members to return to the hotel. Quick dry hand sanitizer and special paper for pressing the elevator button were placed at the entrance to each elevator on each floor.

\subsection{Cleaning and Disinfection of Public Areas of the Hotel}

Hotel public areas are places with a high level of traffic of persons, and thus can be areas where there is a high incidence of cross-infection. Public areas include entrance and exit accesses at the hotel gate, corridors, elevators and stairs on each floor, meeting rooms, and dining rooms. These areas were cleaned and disinfected by the hotel staff every day. The floor was cleaned daily using the mop moistened with 250-500 $\mathrm{mg} / \mathrm{L}$ of a chlorine-containing disinfectant, and then after 30 minutes the floor was wiped with a mop moistened with clean water, and then followed by dry mopping. The 
countertops, elevators, buttons, and door handles were wiped and disinfected with $75 \%$ alcohol or hydrogen peroxide disinfection wipes. The corridors, stairways, and open spaces at the entrances of hotel were disinfected by spraying 3 times a day with a disinfectant containing $500 \mathrm{mg} / \mathrm{L}$ of a chlorine-containing solution that was used immediately after it was diluted, and not reused [4]. Quick-dry hand disinfectants were placed at the entrances of each hotel corridor, the elevators, dining areas, and rooms on each floor.

\subsection{Cleaning and Disinfection of Vehicles}

The distance between the hotel and the hospital was not long, and team members were transported to and from the hospital in special, designated vehicles. The infection and control team were responsible for daily cleaning and disinfection of the interior of the vehicles. The vehicle interiors were wiped once a day with $500 \mathrm{mg} / \mathrm{L}$ chlorine disinfectant solution, and after 30 minutes were wiped again with clean water. The vehicle windows were kept open for ventilation.

\subsection{Room Partitioning}

Hotel rooms were divided into a non-clean area, buffer zone, decontamination area, and clean area. The area outside the door and the area immediately after entering the room were the non-clean area. The toilet was the decontamination area, and area between the bar and the sofa was clean living area (Figure 1). Two or 3 hooks for hanging clothes were placed in the non-clean area, which also contained disposable gloves, hands-free decontamination solution, disinfectant wipes, and trash cans. (2) The decontamination area (toilet), contained a laundry tub, hand sanitizer, 84 disinfectant or chlorine-containing disinfection tablets, 75\% alcohol, iodophor, saline, and alcohol.

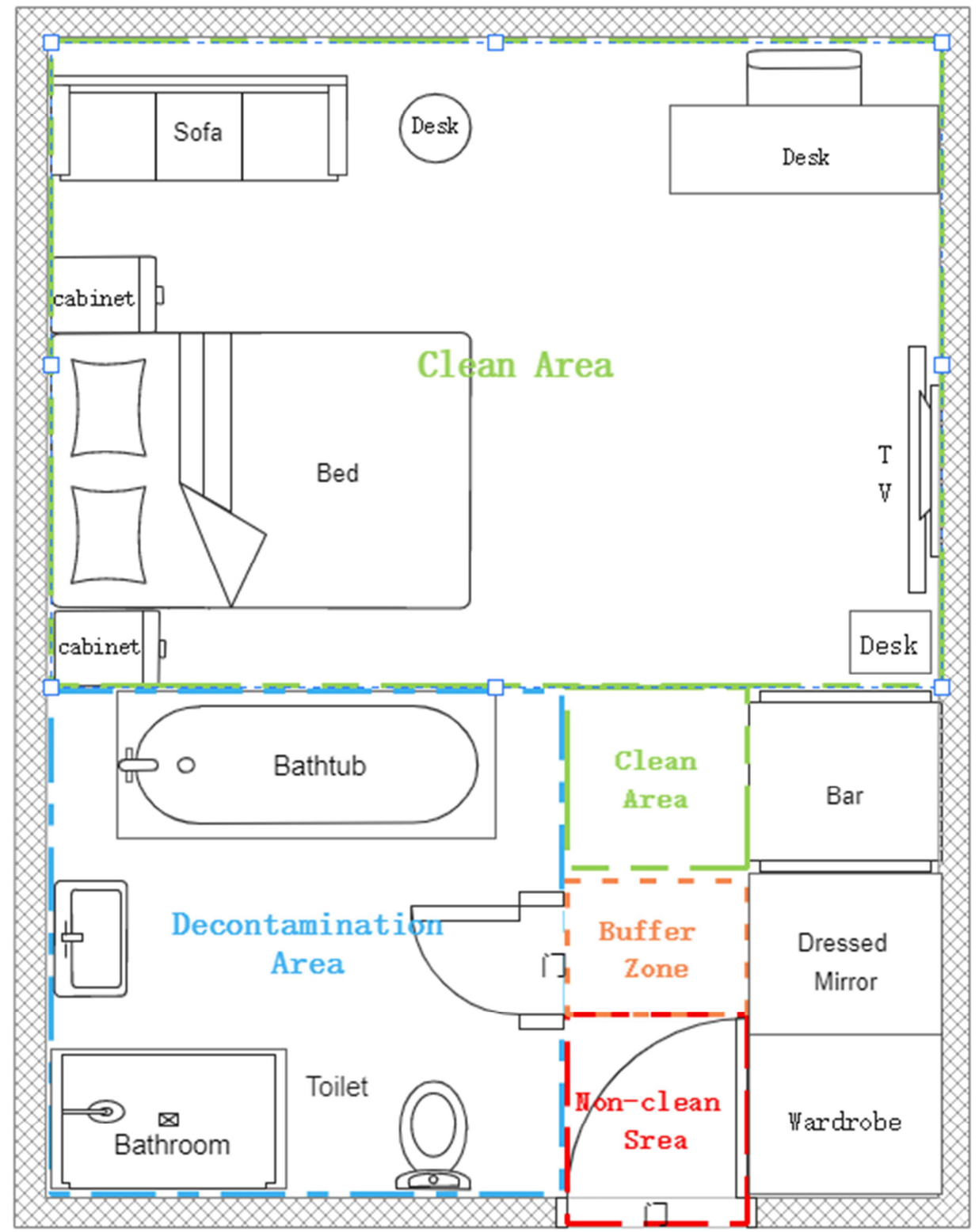

Figure 1. Layout of hotel rooms in Wuhan used by the medical support team. 


\section{Hotel Process Management}

\subsection{Hotel Exit Process}

The process of leaving the hotel consisted of a number of defined steps. 1) Clean clothes were prepared and placed in the decontamination area, so that team members could change into clean clothes after personal cleaning. 2) Specific clothes and shoes were worn for travel to the hospital, and members wore a surgical mask and carried an extra one. 3) Members exited the hotel via the clean elevators and through the designated exit. Quick-drying hand disinfectant was used immediately after pressing the elevator button, or paper was used to press the button. 4) Members were transported to the hospital in a designated vehicle. They entered the hospital through a "clean" medical staff entrance and immediately went to a specific room to change their clothes and shoes. 5) Protective equipment was worn before entering any potentially contaminated area [5].

\subsection{Process of Returning to the Hotel After Work}

Similar to leaving the hotel, returning to the hotel was composed of a number of specific steps. 1) Protective equipment was removed according to an established protocol in the CoVID-19 ward, followed by hand washing, wearing a medical surgical mask, and leaving the hospital [1]. 2) After arriving at the designated hotel entrance, hands were disinfected with an alcohol-containing quick-drying hand disinfectant, followed by taking off the surgical mask and discarding it in a special garbage bin. 3) Hands were disinfected again, and a new surgical mask was worn. 4) The soles of the feet were disinfected by stepping on the disinfectant foot pads, followed by receiving a body temperature measurement, entering the hotel, and taking a clean elevator to return to the room. A piece of paper was used to press the elevator button, or hands were disinfected with a quick-drying hand disinfectant if the paper was not used.

\subsection{Process of Entering the Hotel Room}

\subsubsection{Procedures in Non-clean Areas}

Procedures in non-clean areas are: 1) Hands were sanitized, followed by putting on gloves. 2) Hydrogen peroxide disinfectant wipes were used to wipe the front of the clothes. 3) After taking off the pants and hanging them on the outside of the door, disinfectant wipes were used to wipe the back of the clothes for use the next day. 4) Disinfectant wipes were used to wipe the feet. 5) After taking off the gloves, hand hygiene was performed followed by opening the door and changing into slippers in the non-clean area of the room.

\subsubsection{Processes in the Decontamination Area}

Processes in the decontamination area included: 1) After entering the decontamination area, hands were washed with a sanitizer under running water. (2) Clothes were removed and placed in a designated bin. The external ear canal, nasal cavity, and eyes were cleaned by washing. It is not recommended to routinely use disinfectants such as alcohol or iodophor to disinfect mucous membranes, such as the nasal cavity, oral cavity, and external auditory meatus. If there was a suspicion of contamination or exposure, and a sufficient treatment was not performed at the hospital, and the mucosa were washed with $0.05 \%$ iodophor or saline solution [6,7]. 3) Clean clothes and slipper were put on before entering the clean living area. 4) Clothes for going to hospital, and clothes for staying in the hotel clean living area were cleaned separately $[6,8]$.

\subsection{Dining Process}

Specific processes were put in place for meals. 1) Medical staff did not dine together. The hotel staff prepared individual meals for breakfast, lunch, and dinner and team members ate in their rooms. 2) Team members were required to take a bath and wear the clean clothes before going to the hotel restaurant for a meal. 3) Surgical masks were worn in the restaurant, and hand sanitizer was used before entering the restaurant. 4) The number of persons in the restaurant at any time was limited, persons sat at an angle to each other to avoid face-to-face contact, and maintained a minimum distance of 1 meter from other people [9]. When the mask was removed to eat, it was folded and wrapped inside of a piece of paper to avoid putting it directly on the dining table.

\section{Team Member Behavior Management}

1) Each team member was provided their own room to reduce the risk of cross infection, and to avoid interference from each other. 2) Members were not allowed to travel outside of the hotel, and if travel was necessary they reported it to the team leader. 3) Meeting local relatives and friends was not allowed. 4) A mask, shoes, and socks (no slippers) were required when engaging in activities in public areas of the hotel (e.g., meetings). 5) Face-to-face meetings were avoided whenever possible. Members were not allowed to have an entertaining gathering and face to face visiting should be reduced. 6) When talking to others in person, a minimum distance of 1 meter was required and shaking hands was not allowed. 7) Team members were instructed to keep warm, drink plenty of water, and dry hair completely before leaving the hotel. 8) Members ate an egg, drank a glass of milk, and consumed fresh fruits and vegetables daily, as well as exercising daily in the hotel room to improve immunity. 9) The hotel room windows were opened for ventilation for 2 to 3 times a day, $1 / 2$ hour each time. The central air-conditioning should be turned on by a professional manager, but the independent air-conditioning could be turned on by the team member themselves. 10) Team members cleaned their own rooms. The floor was wiped with a disinfectant containing $500 \mathrm{mg} / \mathrm{L}$ of available chlorine every day for 30 minutes, followed by wiping with clean water. Hydrogen peroxide disinfectant wipes or $75 \%$ alcohol were used to wipe surfaces, especially high-contact areas 
such as the bathroom, door handles, switches, tables, and mobile phones. Chlorine-containing disinfectants were not used for this due to their strong corrosiveness and long action time. When using alcohol, attention was paid to the presence of open flames [4]. Team members were required to monitor their body temperature and the presence of any physical symptoms, and assess their own psychological status and sleep status daily, and report the information to the infection control group through the Jinshan document applet. 12) Each team member was provided a portable dynamic electrocardiogram device so they could check their heart rhythm daily. Any symptoms such as chest discomfort or breathing difficulties were to be reported to the infection control team.

\section{Health Management of Team Members}

\subsection{Self-temperature and Physical Symptom Monitoring}

All team members monitored their own body temperature, physical symptoms, psychological status, and sleep status. In a member had a fever (body temperature $>37.3^{\circ} \mathrm{C}$ ) or typical symptoms of NCP such as dry cough, chest tightness, difficulty breathing, fatigue, diarrhea (excluding an identifiable cause such as diet or stress), or insomnia they were required to report the information to the infection control team. The infection control team then decided if evaluation by an infection control expert or psychologist was needed. Members with a risk of infection were isolated to their room.

\subsection{Occurrence of Occupational Exposure}

If a team member had occupational exposure in the work area, they were treated based on specific methods for high or low risk exposure. The infection control team leader reported the exposure to the hospital's infection control department, and the head of the department determined the severity of occupational exposure and subsequent treatment.

\subsection{Team Member Psychological Well-being}

The team leader, medical team leader and nursing team leader communicated more with the team members, gave active concern for the team members in various inspects to understand their psychological state and perform mental and stress relief.

Each week team members completed a questionnaire regarding their psychological well-being, and was assessed by the medical team. Members with relatively high anxiety and depression scores, and/or insomnia received personal psychological counseling by a certified psychologist. When necessary, appropriate rest was allowed, or medications were prescribed $[10,11]$.

\subsection{Health Record for the Team Members}

The members who were unwell and left the warehouse early were followed up to record their health status daily.
Special attention was paid to their psychological status, body temperature, and respiratory and digestive symptoms, until their symptoms had disappeared for 3 days.

\subsection{Team Member Nutrition}

Three nutritious meals a day were provided. Meals consisted of meats, fresh fruits and vegetables, milk and eggs, and moderate amounts of potassium-rich foods, which were supplied 24 hours, so that the night duty members could have nutritious meals.

\subsection{Shift Scheduling}

Work was scheduled as 4 shifts per day. In this manner, team members worked 6 hours a day. This allowed members to have adequate time for rest.

\section{Conclusions}

In the face of the COVID-19 public health emergency, the medical team of Guangdong Province protected the members from infection through establishment of an infection and control team, infection and control knowledge training, and standardized protocols for disinfection, assess, personnel behavior, and health management. However, we found that many members of the medical team had anxiety, depression, insomnia, and other phenomena. Thus, we recommend that medical staff should receive psychological crisis intervention training in advance so they can understand responses to stress, and learn how to respond to the stress and regulate emotions [12-15]. It is necessary to provide psychological counseling for the medical staff. Through professional psychological counseling, venting emotions, and discovering beautiful things around themselves $[11,16]$, the medical staff will have more courage and confidence to fight the epidemic with a scientific and positive attitude.

\section{References}

[1] Zhu N., Zhang D., Wang W., et al. (2019). China Novel Coronavirus Investigating and Research Team. A novel coronavirus from patients with pneumonia in China [J]. N Engl J Med. 382 (8): 727-733.

[2] Adhikari SP, Meng S, Wu YJ, et al. (2020). Epidemiology, causes, clinical manifestation and diagnosis, prevention and control of coronavirus disease (COVID-19) during the early outbreak period: a scoping review [J]. Infect Dis Poverty. 9 (1): 29-29.

[3] Lu J. J., Si X., Yi H., et al. (2020). Problems and coping strategies for control of infection in individuals and residences of medical teams giving support to Wuhan during COVID-19 epidemic [J]. Chin J Nosocomiol. 30 (10): 1-5.

[4] Novel coronavirus pneumonia prevention and control plan issued by the general office of the national health and Health Committee of People's Republic of China (Fifth Edition) [EB/OL]. [2020-04-13]. http://www.nhc.gov.cn/jkj/s3577/202002/a5d6f7b8c48c451c8 7dba14889b30147.shtml. 
[5] Novel coronavirus pneumonia Protection Office Technical Guidelines for medical workers during the new crown pneumonia outbreak (Trial) [EB/OL]. (2020-02-21) [2020-04-13]. http://www.nhc.gov.cn/yzygj/s7659/202001/e-71c5de925a64eafb elce790debab5c6.shtml.

[6] Li L. Y., Wu A. H., Yao X. (2020). Prevention and control of novel coronavirus pneumonia in resident of medical team [J]. Chin J Infect Control, 19 (02): 123-125.

[7] Ma P., Liu X. P., Ma H. (2003). Harm and correct use of disinfectants during SARS [J]. Chin J Public Health, (09): 127.

[8] Zhu S. C., Qiao F., Luo F. M., et al. (2020). Recommendations on infection prevention and control of resident from medical team for aiding Hubei of West China Hospital of Sichuan University in the period of coronavirus disease 2019 outbreak [J]. West China Medical Journal, 35 (03): 255-260.

[9] Li L. Y., Wu A. H. (2020). Confusion on prevention and control of healthcare-associated infection of novel coronavirus $[\mathrm{J}]$. Chin J Infect Control, 19 (02): 105-108.

[10] Yu W. L., Sun D. Y. (2020). Intervention of mental health risks of female nurses engaged in nursing patients with COVID-19. Occup Health \& Emerg Rescue, 38 (02): 106-108.
[11] Chen Q, Liang M, Li Y, et al. (2020). Mental health care for medical staff in China during the COVID-19 outbreak [J]. Lancet Psychiatry. 7 (4): e15-e16.

[12] Li J, Yang Z, Qiu H, et al. (2020). Anxiety and depression among general population in China at the peak of the COVID-19 epidemic. World Psychiatry. 19 (2): 249-250.

[13] Rajkumar RP. (2020). COVID-19 and mental health: A review of the existing literature. Asian J Psychiatr. 52: 102066.

[14] Liu Q, Luo D, Haase JE, et al. (2020). The experiences of health-care providers during the COVID-19 crisis in China: a qualitative study. Lancet Glob Health. 8 (6): e790-e798.

[15] Ming Y. H., Mi Y. Y., Nie T., et al. (2020). Establishment of Management System for COVID-19 Prevention in Intensive Care Unit and Its Effect [J]. Journal of Nursing (China), 27 (05): 55-59.

[16] Feng M., Zhang Y. L., Song Z. F., et al. (2020). Diagnosis and treatment of novel coronavirus pneumonia in West China Hospital: internal management of Wuhan Medical Center [J]. Chinese Journal of Respiratory and Critical Care Medicine, 19 (02): 132-133. 